\title{
Non-negative global weak solutions for a degenerate parabolic system modelling thin films driven by capillarity
}

\author{
Bogdan-Vasile Matioc \\ Institut für Angewandte Mathematik, Leibniz Universität Hannover, \\ Welfengarten 1, 30167 Hannover, Germany \\ (matioc@ifam.uni-hannover.de)
}

(MS received 19 April 2011; accepted 19 October 2011)

\begin{abstract}
We prove the global existence of non-negative weak solutions for a strongly coupled, fourth-order degenerate parabolic system governing the motion of two thin fluid layers in a porous medium when capillarity is the sole driving mechanism.
\end{abstract}

\section{Introduction and the main result}

In this paper we study the following one-dimensional degenerate system of equations:

$$
\left.\begin{array}{l}
\partial_{t} f=-\partial_{x}\left[f \partial_{x}^{3}(A f+B g)\right], \\
\partial_{t} g=-\partial_{x}\left[g \partial_{x}^{3}(f+g)\right],
\end{array}\right\} \quad(t, x) \in(0, \infty) \times(0, L),
$$

which models the dynamics of two thin fluid threads in a porous medium in the absence of gravity. One of the fluids is located in the region bounded from below by the line $y=0$ and from above by the graph $y=f(t, x)$, while the region occupied by the second fluid is located between the graphs $y=f(t, x)$ and $y=(f+g)(t, x)$, $f$ and $g$ being non-negative functions. Furthermore, $L$ is a positive real number and the positive constants $A$ and $B$ have the following physical meaning:

$$
A:=\frac{\mu_{+}}{\mu_{-}} \frac{\gamma_{d}+\gamma_{w}}{\gamma_{d}}>B:=\frac{\mu_{+}}{\mu_{-}} .
$$

We let $\mu_{-}\left(\mu_{+}\right)$denote the viscosity of the fluid located below (above), $\gamma_{w}$ is the surface tension coefficient at the interface $y=f(t, x)$ between the wetting phases, while $\gamma_{d}$ is the surface tension coefficient at the interface $y=(f+g)(t, x)$. The system (2.1) is supplemented by initial conditions

$$
f(0)=f_{0}, \quad g(0)=g_{0}, \quad x \in(0, L),
$$

whereby $f_{0}$ and $g_{0}$ are assumed to be known, and we impose no-flux boundary conditions

$$
\partial_{x} f=\partial_{x} g=\partial_{x}^{3} f=\partial_{x}^{3} g=0, \quad x=0, L .
$$

The system (1.1) was obtained in [7], by passing to the small layer thickness in the Muskat problem studied in [8]. This is a widely used approach in the study

(C) 2012 The Royal Society of Edinburgh 
of thin fluid threads because it reduces complex moving boundary-value problems to local problems defined by generally simpler equations. System (1.1) is strongly related to the thin film equation because if, for instance, $f$ is constantly equal to zero, then $g$ is a solution of the thin film equation

$$
\partial_{t} g+\partial_{x}\left(g^{n} \partial_{x}^{3} g\right)=0
$$

when $n=1$. We refer the reader to the survey papers [1,10], in which many aspects concerning the thin film equation are discussed. It should be noted that similar methods to those in $[7]$ have been used in $[9,11]$ to rigorously show that, in the limit of thin fluid threads, the solutions of the moving boundary-value problems for Stokes and Hele-Shaw flows converge towards the corresponding solutions (determined by the initial data) of the thin film equation (1.4), with $n=3$ for Stokes and $n=1$ for the Hele-Shaw flow. Compared with the thin film equation, system (1.1) is more involved because it is strongly coupled, both equations of (1.1) containing highestorder derivatives of $f$ and $g$, and, furthermore, there are two sources of degeneracy, because both $f$ and $g$ may equal zero. Since both equations in (1.1) have fourth order, we cannot rely on maximum principles when studying problem (1.1).

Corresponding to (1.1), we define the following energy functionals:

$$
\mathcal{E}_{1}(f, g):=\frac{1}{2} \int_{0}^{L}\left|\partial_{x} f\right|^{2}+\frac{B}{A-B}\left|\partial_{x}(f+g)\right|^{2} \mathrm{~d} x
$$

and

$$
\mathcal{E}_{2}(f, g):=\int_{0}^{L} \Phi(f)+B \Phi(g) \mathrm{d} x
$$

whereby the function $\Phi$ is given by $\Phi(s):=s \ln (s)-s+1$ for all $s \geqslant 0$. They will play a key role when constructing the weak solutions for the problem (1.1)-(1.3).

Using these two functionals and Galerkin approximations, we prove that the problem (1.1)-(1.3) possesses, for non-negative initial data, non-negative global weak solutions. To this end, we first regularize system (1.1) and use the functional $\mathcal{E}_{2}$ to establish convergence of certain Galerkin approximations towards global weak solutions (of the regularized problem) which satisfy similar energy estimates to the classical solutions of (1.1)-(1.3). In a second step, we show that weak solutions of the regularized problem converge towards non-negative global weak solutions of the original system (1.1). The uniqueness of our weak solutions is left as an open problem (this is still an open problem also for the thin film equation; cf. $[2,5]$ ). We note that it has only recently been shown in [14] (see also $[3,4]$ ), in the context of the thin film equation, that the non-negative weak solutions found in [2] converge exponentially fast in $H^{1}$ towards flat equilibria. In our case, this is a further open question. The second-order version of (1.1), when the fluids are driven only by gravity and surface tension is neglected, has been recently investigated in [6], where the existence of non-negative global weak solutions which converge exponentially fast in $L_{2}$ to flat equilibria is established (see also [7]).

In order to state our main result, we now introduce the function spaces we work with. For each $m \in \mathbb{N}$, we let $H^{m}:=H^{m}((0, L))$ be the $L_{2}$-based Sobolev space and we let $H_{\Delta}^{m}$ denote the closed subspace of $H^{m}$ which has $\left\{\phi_{k}: k \in \mathbb{N}\right\}$ as its basis. 
Herein,

$$
\phi_{0}:=\sqrt{\frac{1}{L}} \quad \text { and } \quad \phi_{k}:=\sqrt{\frac{2}{L}} \cos \left(\frac{k \pi x}{L}\right), \quad k \geqslant 1,
$$

are the normalized eigenvectors of the operator $-\partial_{x}^{2}: H^{2} \rightarrow L_{2}$ with zero Neumann boundary conditions. To be more precise, $f \in H_{\Delta}^{m}$ if and only if the Fourier series associated to $f$ converges towards $f$ in $H^{m}$. It is well known that $H_{\Delta}^{1}=H^{1}$ and it is not difficult to see that, for $m \geqslant 4$, the boundary conditions (1.3) are satisfied by functions from this space.

Given $T \in(0, \infty]$, let $Q_{T}:=(0, T) \times(0, L)$. The main result of this paper is the following theorem.

THEOREM 1.1. Let $f_{0}, g_{0} \in H^{1}$ be two non-negative functions. There exists a global weak solution $(f, g)$ of $(1.1)$ with $(f(0), g(0))=\left(f_{0}, g_{0}\right)$ which has the following properties:

(i) $f \geqslant 0$ and $g \geqslant 0$ in $(0, T) \times(0, L)$;

(ii) $f, g \in L_{\infty}\left(0, T ; H^{1}\right) \cap L_{2}\left(0, T ; H_{\Delta}^{2}\right) \cap C\left([0, T], C^{\alpha}([0, L])\right)$ for some arbitrary $\alpha \in\left(0, \frac{1}{2}\right)$ and $\sqrt{f} \partial_{x}^{3}(A f+B g), \sqrt{g} \partial_{x}^{3}(f+g) \in L_{2}\left(Q_{T}^{+}\right)$, where

$$
Q_{T}^{+}:=\left\{(t, x) \in Q_{T}:(f g)(t, x)>0\right\}
$$

(iii)

$$
\begin{array}{r}
\int_{0}^{L} f(T) \psi \mathrm{d} x-\int_{0}^{L} f_{0} \psi \mathrm{d} x+\int_{Q_{T}}\left(A \partial_{x}^{2} f+B \partial_{x}^{2} g\right)\left(\partial_{x} f \partial_{x} \psi+f \partial_{x}^{2} \psi\right) \mathrm{d} x \mathrm{~d} t=0 \\
\int_{0}^{L} f(T) \psi \mathrm{d} x-\int_{0}^{L} f_{0} \psi \mathrm{d} x+\int_{Q_{T}}\left(\partial_{x}^{2} f+\partial_{x}^{2} g\right)\left(\partial_{x} g \partial_{x} \psi+g \partial_{x}^{2} \psi\right) \mathrm{d} x \mathrm{~d} t=0
\end{array}
$$

for all $T>0$ and $\psi \in H_{\Delta}^{2}$; furthermore, the weak solutions satisfy

(iv) $\|f(T)\|_{L_{1}}=\left\|f_{0}\right\|_{L_{1}}$ and $\|g(T)\|_{L_{1}}=\left\|g_{0}\right\|_{L_{1}}$,

(v) $\mathcal{E}_{2}(f(T), g(T))+\int_{Q_{T}}(A-B)\left|\partial_{x}^{2} f_{\varepsilon}\right|^{2}+B\left|\partial_{x}^{2}\left(f_{\varepsilon}+g_{\varepsilon}\right)\right|^{2} \mathrm{~d} x \mathrm{~d} t \leqslant \mathcal{E}_{2}\left(f_{0}, g_{0}\right)$

for all $T \in(0, \infty)$; and

(vi) $\mathcal{E}_{1}(f(T), g(T))+\int_{Q_{T}^{+}} f\left|\partial_{x}^{3}(A f+B g)\right|^{2}+B g\left|\partial_{x}^{3}(f+g)\right|^{2} \mathrm{~d} x \mathrm{~d} t \leqslant \mathcal{E}_{1}\left(f_{0}, g_{0}\right)$

for almost all $T \in(0, \infty)$.

We note that since $f(t)$ and $g(t)$ belong to $H_{\Delta}^{2}$ for almost all $t>0$, they satisfy the homogeneous Neumann boundary conditions at $x=0$ and $x=L$ for all such $t$.

The outline of the paper is as follows: in $\S 2$ we introduce a regularized version of (1.1) and use Galerkin approximations to find, in the limit, global weak solutions of this regularized problem (see proposition 2.1). Introducing the regularized system allows us on the one hand to use the energy functional $\mathcal{E}_{1}$ when dealing with the 
Galerkin approximations, and, on the other hand, to control the solutions of the regularized problem when they become negative. In $\S 3$ we show, by combining energy estimates for both functionals $\mathcal{E}_{1}$ and $\mathcal{E}_{2}$, that the weak solutions of the regularized problem converge towards non-negative global weak solutions of our original problem (1.1)-(1.3).

\section{The regularized system}

In order to prove theorem 1.1 we shall regularize system (1.1) and use Galerkin approximations to build global weak solutions for this regularized problem. In $\S 3$ these solutions are shown to converge towards weak solutions of (1.1). To this end, given $\varepsilon \in(0,1]$, we define the Lipschitz continuous function $a_{\varepsilon}: \mathbb{R} \rightarrow \mathbb{R}$ by the relation

$$
a_{\varepsilon}(s):= \begin{cases}s+\varepsilon, & s \geqslant 0 \\ \varepsilon, & s<0 .\end{cases}
$$

Furthermore, we define the convex function $\Phi_{\varepsilon}: \mathbb{R} \rightarrow \mathbb{R}$ by

$$
\Phi_{\varepsilon}(s):= \begin{cases}(s+\varepsilon) \ln (s+\varepsilon)-(s+\varepsilon)+1, \quad s \geqslant 0 \\ \frac{s^{2}}{2 \varepsilon}+s \ln (\varepsilon)+\varepsilon \ln (\varepsilon)-\varepsilon+1, \quad s<0 .\end{cases}
$$

Since we choose $\varepsilon \leqslant 1$, it is easy to see that $\Phi_{\varepsilon}(s) \geqslant 0$ for all $s \in \mathbb{R}$ and that $\Phi_{\varepsilon}^{\prime \prime}=1 / a_{\varepsilon}$. With this notation, we introduce the following regularized version of our original problem (1.1):

$$
\left.\begin{array}{l}
\partial_{t} f_{\varepsilon}=-\partial_{x}\left[a_{\varepsilon}\left(f_{\varepsilon}\right) \partial_{x}^{3}\left(A f_{\varepsilon}+B g_{\varepsilon}\right)\right], \\
\partial_{t} g_{\varepsilon}=-\partial_{x}\left[a_{\varepsilon}\left(g_{\varepsilon}\right) \partial_{x}^{3}\left(f_{\varepsilon}+g_{\varepsilon}\right)\right],
\end{array}\right\} \quad(t, x) \in(0, \infty) \times(0, L)
$$

Of course, this system is coupled with the initial and boundary conditions (1.2) and (1.3). Compared with (1.1), the only difference is that we have replaced $f$ and $g$ in (1.1) by $a_{\varepsilon}(f)$ and $a_{\varepsilon}(g)$, respectively, and controlled in this way the functions $f_{\varepsilon}, g_{\varepsilon}$ when they take negative values (see the definition of $a_{\varepsilon}$ ). Furthermore, by choosing the regularization in this way, we may still use the functional $\mathcal{E}_{1}$ to obtain useful estimates for the solutions of (2.3). For the problem consisting of (2.3) and (1.2), (1.3), we prove the following result.

Proposition 2.1. Let $f_{0}, g_{0} \in H^{1}$ be two non-negative functions and $\varepsilon \in(0,1]$. There exist globally defined functions $f_{\varepsilon}$ and $g_{\varepsilon}$ with $f_{\varepsilon}(0)=f_{0}, g_{\varepsilon}(0)=g_{0}$, which have the following properties.

(i) Given $T>0$, the functions

$$
f_{\varepsilon}, g_{\varepsilon} \in L_{\infty}\left([0, T], H^{1}\right) \cap L_{2}\left(0, T ; H_{\Delta}^{3}\right) \cap C\left([0, T], C^{\alpha}([0, L])\right)
$$

for some arbitrary $\alpha \in\left(0, \frac{1}{2}\right)$. 
(ii) For all $T>0$ and $\psi \in H^{1}$ we have

$$
\begin{aligned}
& \int_{0}^{L} f_{\varepsilon}(T) \psi \mathrm{d} x-\int_{0}^{L} f_{0} \psi \mathrm{d} x=\int_{Q_{T}} a_{\varepsilon}\left(f_{\varepsilon}\right) \partial_{x}^{3}\left(A f_{\varepsilon}+B g_{\varepsilon}\right) \partial_{x} \psi \mathrm{d} x \mathrm{~d} t \\
& \int_{0}^{L} g_{\varepsilon}(T) \psi \mathrm{d} x-\int_{0}^{L} g_{0} \psi \mathrm{d} x=\int_{Q_{T}} a_{\varepsilon}\left(g_{\varepsilon}\right) \partial_{x}^{3}\left(f_{\varepsilon}+g_{\varepsilon}\right) \partial_{x} \psi \mathrm{d} x \mathrm{~d} t
\end{aligned}
$$

(iii) The following energy estimates are satisfied:

$$
\begin{gathered}
\left\|f_{\varepsilon}(T)\right\|_{L_{1}}=\left\|f_{0}\right\|_{L_{1}} \quad \text { and } \quad\left\|g_{\varepsilon}(T)\right\|_{L_{1}}=\left\|g_{0}\right\|_{L_{1}}, \\
\int_{0}^{L} \Phi_{\varepsilon}\left(f_{\varepsilon}(T)\right)+B \Phi_{\varepsilon}\left(g_{\varepsilon}(T)\right) \mathrm{d} x \\
+\int_{Q_{T}}(A-B)\left|\partial_{x}^{2} f_{\varepsilon}\right|^{2}+B\left|\partial_{x}^{2}\left(f_{\varepsilon}+g_{\varepsilon}\right)\right|^{2} \mathrm{~d} x \mathrm{~d} t \\
\leqslant \int_{0}^{L} \Phi_{\varepsilon}\left(f_{0}\right)+B \Phi_{\varepsilon}\left(g_{0}\right) \mathrm{d} x \quad \text { for all } T \in[0, \infty)
\end{gathered}
$$

and

$$
\begin{aligned}
& \mathcal{E}_{1}\left(f_{\varepsilon}(T), g_{\varepsilon}(T)\right) \\
& +\frac{1}{A-B} \int_{Q_{T}} a_{\varepsilon}\left(f_{\varepsilon}\right)\left|\partial_{x}^{3}\left(A f_{\varepsilon}+B g_{\varepsilon}\right)\right|^{2}+B a_{\varepsilon}\left(g_{\varepsilon}\right)\left|\partial_{x}^{3}\left(f_{\varepsilon}+g_{\varepsilon}\right)\right|^{2} \mathrm{~d} x \mathrm{~d} t \\
& \quad \leqslant \mathcal{E}_{1}\left(f_{0}, g_{0}\right) \quad \text { for almost all } T \in(0, \infty) .
\end{aligned}
$$

We shall construct the global solutions of (2.3) by using Galerkin's method. In a first step we shall find, by using the Picard-Lindelöf theorem, Galerkin approximations for the solutions of (2.3) which are defined on a positive time interval. Using the energy functional $\mathcal{E}_{1}$, we show then that in fact the approximations are defined globally. In a second step, we prove that the Galerkin approximation converges towards global solutions of the regularized system which satisfy energy inequalities for both energy functionals $\mathcal{E}_{1}$ and $\mathcal{E}_{2}$. Although $f_{0}$ and $g_{0}$ are non-negative, it is not clear if $f_{\varepsilon}$ and $g_{\varepsilon}$ preserve this property in time. However, we shall show in $\S 3$ that, for $\varepsilon \rightarrow 0, f_{\varepsilon}$ and $g_{\varepsilon}$ converge uniformly to non-negative functions.

\subsection{Global existence of the Galerkin approximations}

Given $f_{0}, g_{0} \in H^{1}$, the initial conditions of (1.1), we consider their expansions

$$
f_{0}=\sum_{k=0}^{\infty} f_{0 k} \phi_{k}, \quad g_{0}=\sum_{k=0}^{\infty} g_{0 k} \phi_{k} \quad \text { in } H^{1},
$$

and, for each $n \in \mathbb{N}$, the partial sums

$$
f_{0}^{n}:=\sum_{k=0}^{n} f_{0 k} \phi_{k}, \quad g_{0}^{n}:=\sum_{k=0}^{n} g_{0 k} \phi_{k} .
$$


We first seek the continuously differentiable functions

$$
f_{\varepsilon}^{n}:=\sum_{k=0}^{n} F_{\varepsilon}^{k}(t) \phi_{k}, \quad g_{\varepsilon}^{n}:=\sum_{k=0}^{n} G_{\varepsilon}^{k}(t) \phi_{k}
$$

which solve (2.3) when testing with functions from the vector space $\left\langle\phi_{0}, \ldots, \phi_{n}\right\rangle$, and additionally

$$
f_{\varepsilon}^{n}(0)=f_{0}^{n}, \quad g_{\varepsilon}^{n}(0)=g_{0}^{n} .
$$

By construction, the functions $\left(f_{\varepsilon}^{n}, g_{\varepsilon}^{n}\right)$ satisfy the boundary conditions (1.3) and if we test (2.3) with constant functions, it follows at once that necessarily $F_{\varepsilon}^{0}$ and $G_{\varepsilon}^{0}$ are constant functions

$$
F_{\varepsilon}^{0}(t)=f_{00}, \quad G_{\varepsilon}^{0}(t)=g_{00}, \quad t \geqslant 0 .
$$

Moreover, the tuple $\left(\boldsymbol{F}_{\varepsilon}^{n}, \boldsymbol{G}_{\varepsilon}^{n}\right):=\left(F_{\varepsilon}^{1}, \ldots, F_{\varepsilon}^{n}, G_{\varepsilon}^{1}, \ldots, G_{\varepsilon}^{n}\right)$ is the solution of the initial-value problem

$$
\left(\boldsymbol{F}_{\varepsilon}^{n}, \boldsymbol{G}_{\varepsilon}^{n}\right)^{\prime}=\Psi\left(\boldsymbol{F}_{\varepsilon}^{n}, \boldsymbol{G}_{\varepsilon}^{n}\right), \quad\left(\boldsymbol{F}_{\varepsilon}^{n}, \boldsymbol{G}_{\varepsilon}^{n}\right)(0)=\left(f_{01}, \ldots, f_{0 n}, g_{01}, \ldots, g_{0 n}\right),
$$

where $\Psi:=\left(\Psi_{1}, \Psi_{2}\right): \mathbb{R}^{2 n} \rightarrow \mathbb{R}^{2 n}$ is given by

$$
\begin{aligned}
& \Psi_{1, j}(x, y)=\sum_{k=1}^{n}\left(A x_{k}+B y_{k}\right) \int_{0}^{L} a_{\varepsilon}\left(f_{00} \phi_{0}+\sum_{l=1}^{n} x_{l} \phi_{l}\right) \partial_{x}^{3} \phi_{k} \partial_{x} \phi_{j} \mathrm{~d} x \\
& \Psi_{2, j}(x, y)=\sum_{k=1}^{n}\left(x_{k}+y_{k}\right) \int_{0}^{L} a_{\varepsilon}\left(g_{00} \phi_{0}+\sum_{l=1}^{n} y_{l} \phi_{l}\right) \partial_{x}^{3} \phi_{k} \partial_{x} \phi_{j} \mathrm{~d} x
\end{aligned}
$$

for all $x, y \in \mathbb{R}^{n}$. Since $a_{\varepsilon}$ is Lipschitz continuous, we deduce that $\Psi$ is locally Lipschitz continuous on $\mathbb{R}^{2 n}$, and therefore problem (2.6) possesses a unique solution $\left(\boldsymbol{F}_{\varepsilon}^{n}, \boldsymbol{G}_{\varepsilon}^{n}\right)$ defined on a maximal interval $\left[0, T_{\varepsilon}^{n}\right)$. In order to prove that the solution is global, i.e. $T_{\varepsilon}^{n}=\infty$ for all $\varepsilon \in(0,1]$ and $n \in \mathbb{N}$, we make use of the energy functional $\mathcal{E}_{1}$. Indeed, since $\partial_{x}^{2} f_{\varepsilon}^{n}, \partial_{x}^{2} g_{\varepsilon}^{n} \in\left\langle\phi_{0}, \ldots, \phi_{n}\right\rangle$, we may use them as test functions for (2.3). Integrating by parts, we then get the following relation:

$$
\begin{aligned}
& \frac{\mathrm{d}}{\mathrm{d} t} \mathcal{E}_{1}\left(f_{\varepsilon}^{n}, g_{\varepsilon}^{n}\right) \\
& =\frac{1}{A-B} \int_{0}^{L} A \partial_{x} f_{\varepsilon}^{n} \partial_{t}\left(\partial_{x} f_{\varepsilon}^{n}\right)+B \partial_{x} f_{\varepsilon}^{n} \partial_{t}\left(\partial_{x} g_{\varepsilon}^{n}\right) \\
& \quad+B \partial_{x} g_{\varepsilon}^{n} \partial_{t}\left(\partial_{x} f_{\varepsilon}^{n}\right)+B \partial_{x} g_{\varepsilon}^{n} \partial_{t}\left(\partial_{x} g_{\varepsilon}^{n}\right) \mathrm{d} x \\
& =-\frac{1}{A-B} \int_{0}^{L} A \partial_{x}^{2} f_{\varepsilon}^{n} \partial_{t} f_{\varepsilon}^{n}+B\left[\partial_{x}^{2} f_{\varepsilon}^{n} \partial_{t} g_{\varepsilon}^{n} \mathrm{~d} x+\partial_{x}^{2} g_{\varepsilon}^{n} \partial_{t} g_{\varepsilon}^{n}+\partial_{x}^{2} g_{\varepsilon}^{n} \partial_{t} f_{\varepsilon}^{n}\right] \mathrm{d} x \\
& =-\frac{1}{A-B} \int_{0}^{L}\left[A a_{\varepsilon}\left(f_{\varepsilon}^{n}\right) \partial_{x}^{3} f_{\varepsilon}^{n} \partial_{x}^{3}\left(A f_{\varepsilon}^{n}+B g_{\varepsilon}^{n}\right)+B a_{\varepsilon}\left(g_{\varepsilon}^{n}\right) \partial_{x}^{3} f_{\varepsilon}^{n} \partial_{x}^{3}\left(f_{\varepsilon}^{n}+g_{\varepsilon}^{n}\right)\right. \\
& \left.\quad+B a_{\varepsilon}\left(f_{\varepsilon}^{n}\right) \partial_{x}^{3} g_{\varepsilon}^{n} \partial_{x}^{3}\left(A f_{\varepsilon}^{n}+B g_{\varepsilon}^{n}\right)+B a_{\varepsilon}\left(g_{\varepsilon}^{n}\right) \partial_{x}^{3} g_{\varepsilon}^{n} \partial_{x}^{3}\left(f_{\varepsilon}^{n}+g_{\varepsilon}^{n}\right)\right] \mathrm{d} x,
\end{aligned}
$$


and taking into account that $\mathcal{E}_{1}\left(f_{0}^{n}, g_{0}^{n}\right) \leqslant \mathcal{E}_{1}\left(f_{0}, g_{0}\right)$ for all $n \in \mathbb{N}$, we find after integrating with respect to time that

$$
\begin{aligned}
\mathcal{E}_{1}\left(f_{\varepsilon}^{n}(T), g_{\varepsilon}^{n}(T)\right)+\frac{1}{A-B} \int_{Q_{T}} & a_{\varepsilon}\left(f_{\varepsilon}^{n}\right)\left|\partial_{x}^{3}\left(A f_{\varepsilon}^{n}+B g_{\varepsilon}^{n}\right)\right|^{2} \\
& +B a_{\varepsilon}\left(g_{\varepsilon}^{n}\right)\left|\partial_{x}^{3}\left(f_{\varepsilon}^{n}+g_{\varepsilon}^{n}\right)\right|^{2} \mathrm{~d} x \mathrm{~d} t \leqslant \mathcal{E}_{1}\left(f_{0}, g_{0}\right)
\end{aligned}
$$

for all $T>0$. Hence, there exists a positive constant $C$, which is independent of time, such that $\left|\left(\boldsymbol{F}_{\varepsilon}^{n}(T), \boldsymbol{G}_{\varepsilon}^{n}(T)\right)\right|<C$ for all $T<T_{\varepsilon}^{n}$. Together with (2.5), we conclude that for each $n \in \mathbb{N}$ and $\varepsilon \in(0,1]$, the Galerkin approximations $\left(f_{\varepsilon}^{n}, g_{\varepsilon}^{n}\right)$ are defined globally.

\subsection{Convergence of the Galerkin approximations}

Let $T>0$ and $\varepsilon \in(0,1]$ be fixed. From the energy estimate (2.8) we deduce that

$$
\begin{gathered}
\partial_{x} f_{\varepsilon}^{n}, \partial_{x} g_{\varepsilon}^{n} \quad \text { are bounded in } L_{\infty}\left(0, T ; L_{2}\right), \\
\sqrt{a_{\varepsilon}\left(f_{\varepsilon}^{n}\right)} \partial_{x}^{3}\left(A f_{\varepsilon}^{n}+B g_{\varepsilon}^{n}\right), \sqrt{a_{\varepsilon}\left(g_{\varepsilon}^{n}\right)} \partial_{x}^{3}\left(f_{\varepsilon}^{n}+g_{\varepsilon}^{n}\right) \quad \text { are bounded in } L_{2}\left(Q_{T}\right),
\end{gathered}
$$

uniformly in $n \in \mathbb{N}$ and $\varepsilon \in(0,1]$. In view of $a_{\varepsilon} \geqslant \varepsilon$ and $A>B$, we obtain from (2.10) that

$$
\partial_{x}^{3} f_{\varepsilon}^{n}, \partial_{x}^{3} g_{\varepsilon}^{n} \quad \text { are bounded in } L_{2}\left(Q_{T}\right),
$$

uniformly in $n \in \mathbb{N}$. Furthermore, by virtue of (2.5), we see that the mass of both fluids is preserved by the Galerkin approximations

$$
\int_{0}^{L} f_{\varepsilon}^{n}(t) \mathrm{d} x=\left\|f_{0}\right\|_{L_{1}} \quad \text { and } \quad \int_{0}^{L} g_{\varepsilon}^{n}(t) \mathrm{d} x=\left\|g_{0}\right\|_{L_{1}} \quad \text { for all } t \in[0, T] .
$$

Invoking now (2.9), (2.12), and the Poincaré-Wirtinger inequality we conclude that in fact

$$
f_{\varepsilon}^{n}, g_{\varepsilon}^{n} \text { are bounded in } L_{\infty}\left(0, T ; H^{1}\right) \quad \text { uniformly in } \varepsilon \in(0,1] \text { and } n \in \mathbb{N},
$$

while, owing to (2.11) and (2.12), the same inequality implies

$$
f_{\varepsilon}^{n}, g_{\varepsilon}^{n} \text { are bounded in } L_{2}\left(0, T ; H^{3}\right) \text { uniformly in } n .
$$

We consider now the partial derivatives with respect to time, and observe that the first equation of (2.3) can be written in the more compact form $\partial_{t} f_{\varepsilon}^{n}=-\partial_{x} H_{\varepsilon}^{n}$ where, by (2.10), (2.13), and using the embedding $H^{1} \hookrightarrow L_{\infty}$, the right-hand side $H_{\varepsilon}^{n}:=a\left(f_{n}^{\varepsilon}\right)\left(A \partial_{x}^{3} f_{\varepsilon}^{n}+B \partial_{x}^{3} g_{\varepsilon}^{n}\right)$ is bounded in $L_{2}\left(Q_{T}\right)$ uniformly in $\varepsilon$ and $n$. Therefore, given $\zeta \in H^{1}$, we set

$$
\zeta^{n}:=\sum_{k=0}^{n}\left(\zeta \mid \phi_{k}\right) \phi_{k}
$$

and, using integration by parts, obtain

$$
\begin{aligned}
\left|\left(\partial_{t} f_{\varepsilon}^{n}(t) \mid \zeta\right)\right| & =\left|\left(\partial_{t} f_{\varepsilon}^{n}(t) \mid \zeta^{n}\right)\right| \\
& =\left|\left(H_{\varepsilon}^{n}(t) \mid \partial_{x} \zeta_{n}\right)\right| \\
& \leqslant\left\|H_{\varepsilon}^{n}(t)\right\|_{L_{2}}\left\|\zeta^{n}\right\|_{H^{1}} \\
& \leqslant\left\|H_{\varepsilon}^{n}(t)\right\|_{L_{2}}\|\zeta\|_{H^{1}} .
\end{aligned}
$$


This means that

$$
\partial_{t} f_{\varepsilon}^{n}, \partial_{t} g_{\varepsilon}^{n} \text { are bounded in } L_{2}\left(0, T ;\left(H^{1}\right)^{\prime}\right) \quad \text { uniformly in } \varepsilon \text { and } n \text {. }
$$

Gathering (2.13)-(2.15), we obtain from [13, corollary 4], and by making use of the embeddings

$$
H^{1} \stackrel{\text { comp. }}{\longrightarrow} C^{\alpha}([0, L]) \hookrightarrow\left(H^{1}\right)^{\prime} \quad \text { and } \quad H^{3} \stackrel{\text { comp. }}{\longrightarrow} C^{2+\alpha}([0, L]) \hookrightarrow\left(H^{1}\right)^{\prime}
$$

for $\alpha \in\left[0, \frac{1}{2}\right)$, that

$$
f_{\varepsilon}^{n}, g_{\varepsilon}^{n} \text { are relatively compact in } C\left([0, T], C^{\alpha}([0, L])\right) \cap L_{2}\left(0, T ; C^{2+\alpha}([0, L])\right) \text {. }
$$

Hence, for each $\varepsilon \in(0,1]$, there exist functions

$$
f_{\varepsilon}, g_{\varepsilon} \in C\left([0, T], C^{\alpha}([0, L])\right) \cap L_{2}\left(0, T ; C^{2+\alpha}([0, L])\right)
$$

and subsequences of $\left(f_{\varepsilon}^{n}\right)$ and $\left(g_{\varepsilon}^{n}\right)$ (which we denote again by $\left(f_{\varepsilon}^{n}\right)$ and $\left.\left(g_{\varepsilon}^{n}\right)\right)$ such that

$$
f_{\varepsilon}^{n} \rightarrow f_{\varepsilon} \quad \text { and } \quad g_{\varepsilon}^{n} \rightarrow g_{\varepsilon} \quad \text { in } C\left([0, T], C^{\alpha}([0, L])\right) \cap L_{2}\left(0, T ; C^{2+\alpha}([0, L])\right)
$$

Moreover, we deduce from (2.14) that

$$
\partial_{x}^{p} f_{\varepsilon}^{n} \rightarrow \partial_{x}^{p} f_{\varepsilon} \quad \text { and } \quad \partial_{x}^{p} g_{\varepsilon}^{n} \rightarrow \partial_{x}^{p} g_{\varepsilon} \quad \text { in } L_{2}\left(Q_{T}\right) \text { for } p=1,2,3
$$

and therefore $f_{\varepsilon}, g_{\varepsilon} \in L_{2}\left([0, T], H^{3}\right)$. Additionally, since $f_{\varepsilon}^{n}(t), g_{\varepsilon}^{n}(t) \in H_{\Delta}^{3}$, we obtain, by virtue of (2.16), that $\partial_{x} f_{\varepsilon}=\partial_{x} g_{\varepsilon}=0$ at $x=0, L$ for almost all $t \in[0, T]$, which yields $f_{\varepsilon}, g_{\varepsilon} \in L_{2}\left([0, T], H_{\Delta}^{3}\right)$.

\subsection{Proof of proposition 2.1}

First of all, $f_{\varepsilon}^{n}(0)=f_{0}^{n}$ for all $n \in \mathbb{N}$ and since $f_{0} \in H^{1}$ we conclude that $f_{\varepsilon}(0)=f_{0}$ for all $\varepsilon \in(0,1]$. Similarly, we have $g_{\varepsilon}(0)=g_{0}$ for all $\varepsilon \in(0,1]$. Furthermore, it is clear from (2.12) and (2.16) that the weak solutions $\left(f_{\varepsilon}, g_{\varepsilon}\right)$ satisfy $(2.4 a)$.

We pass now to the limit in the energy estimate (2.8). By virtue of (2.10), (2.16) and (2.17) we have

$$
\left.\begin{array}{c}
\sqrt{a_{\varepsilon}\left(f_{\varepsilon}^{n}\right)} \partial_{x}^{3}\left(A f_{\varepsilon}^{n}+B g_{\varepsilon}^{n}\right) \rightarrow \sqrt{a_{\varepsilon}\left(f_{\varepsilon}\right)} \partial_{x}^{3}\left(A f_{\varepsilon}+B g_{\varepsilon}\right), \\
\sqrt{a_{\varepsilon}\left(g_{\varepsilon}^{n}\right)} \partial_{x}^{3}\left(f_{\varepsilon}^{n}+g_{\varepsilon}^{n}\right) \rightarrow \sqrt{a_{\varepsilon}\left(g_{\varepsilon}\right)} \partial_{x}^{3}\left(f_{\varepsilon}+g_{\varepsilon}\right)
\end{array}\right\} \quad \text { in } L_{2}\left(Q_{T}\right)
$$

Furthermore, by (2.16) we know that $f_{\varepsilon}^{n}(t) \rightarrow f_{\varepsilon}(t)$ in $H^{1}$ for almost all $t \in[0, T]$, so that, by passing to the limit $n \rightarrow \infty$ in $(2.8)$, we obtain the estimate $(2.4 c)$.

Claim (i) of proposition 2.1 is now a simple consequence of the assertions $(2.4 \mathrm{a})$ and $(2.4 c)$ in proposition 2.1 (iii).

We now prove assertion (ii) of proposition 2.1. To this end, we pick an arbitrary function $\psi \in H^{1}$ and, testing (2.3) with $\psi^{n}:=\sum_{k=0}^{n}\left(\psi \mid \phi_{k}\right) \phi_{k}$, we obtain the 
following relations:

$$
\begin{aligned}
& \int_{0}^{L} f_{\varepsilon}^{n}(T) \psi^{n} \mathrm{~d} x-\int_{0}^{L} f_{0 n} \psi^{n} \mathrm{~d} x=\int_{Q_{T}} a_{\varepsilon}\left(f_{\varepsilon}^{n}\right) \partial_{x}^{3}\left(A f_{\varepsilon}^{n}+B g_{\varepsilon}^{n}\right) \partial_{x} \psi^{n} \mathrm{~d} x \mathrm{~d} t \\
& \int_{0}^{L} g_{\varepsilon}^{n}(T) \psi^{n} \mathrm{~d} x-\int_{0}^{L} g_{0 n} \psi^{n} \mathrm{~d} x=\int_{Q_{T}} a_{\varepsilon}\left(g_{\varepsilon}^{n}\right) \partial_{x}^{3}\left(f_{\varepsilon}^{n}+g_{\varepsilon}^{n}\right) \partial_{x} \psi^{n} \mathrm{~d} x \mathrm{~d} t .
\end{aligned}
$$

Invoking (2.10) and (2.13), we see that $a_{\varepsilon}\left(f_{\varepsilon}^{n}\right) \partial_{x}^{3}\left(A f_{\varepsilon}^{n}+B g_{\varepsilon}^{n}\right)$ and $a_{\varepsilon}\left(g_{\varepsilon}^{n}\right) \partial_{x}^{3}\left(f_{\varepsilon}^{n}+g_{\varepsilon}^{n}\right)$ are bounded in $L_{2}\left(Q_{T}\right)$ uniformly in $\varepsilon$ and $n$. Using (2.16) and (2.17), we may even identify their weak limit

$$
\left.\begin{array}{c}
a_{\varepsilon}\left(f_{\varepsilon}^{n}\right) \partial_{x}^{3}\left(A f_{\varepsilon}^{n}+B g_{\varepsilon}^{n}\right) \rightarrow a_{\varepsilon}\left(f_{\varepsilon}\right) \partial_{x}^{3}\left(A f_{\varepsilon}+B g_{\varepsilon}\right), \\
a_{\varepsilon}\left(g_{\varepsilon}^{n}\right) \partial_{x}^{3}\left(f_{\varepsilon}^{n}+g_{\varepsilon}^{n}\right) \rightarrow a_{\varepsilon}\left(g_{\varepsilon}\right) \partial_{x}^{3}\left(f_{\varepsilon}+g_{\varepsilon}\right)
\end{array}\right\} \quad \text { in } L_{2}\left(Q_{T}\right)
$$

and assertion (ii) of proposition 2.1 follows from the previous identities when letting $n \rightarrow \infty$.

We end this paragraph with the proof of the estimate $(2.4 b)$. Let us observe that $\Phi_{\varepsilon}^{\prime}\left(f_{\varepsilon}^{n}(t)\right)$ and $\Phi_{\varepsilon}^{\prime}\left(f_{\varepsilon}(t)\right)$ both belong to $H^{1}$ for almost all $t \in[0, T]$, meaning that

$$
\Phi_{\varepsilon}^{\prime}\left(f_{\varepsilon}^{n}(t)\right)=\sum_{k=0}^{\infty}\left(\Phi_{\varepsilon}^{\prime}\left(f_{\varepsilon}^{n}(t)\right) \mid \phi_{k}\right) \phi_{k}, \quad \Phi_{\varepsilon}^{\prime}\left(f_{\varepsilon}(t)\right)=\sum_{k=0}^{\infty}\left(\Phi_{\varepsilon}^{\prime}\left(f_{\varepsilon}(t)\right) \mid \phi_{k}\right) \phi_{k} \quad \text { in } H^{1}
$$

for almost all $t \in[0, T]$. Of course, (2.19) is also valid when replacing $f$ by $g$. In view of (2.19), we obtain the following relation:

$$
\begin{aligned}
\frac{\mathrm{d}}{\mathrm{d} t} \int_{0}^{L} \Phi_{\varepsilon}\left(f_{\varepsilon}^{n}\right)+B \Phi_{\varepsilon}\left(g_{\varepsilon}^{n}\right) \mathrm{d} x= & \int_{0}^{L} \Phi_{\varepsilon}^{\prime}\left(f_{\varepsilon}^{n}\right) \partial_{t} f_{\varepsilon}^{n}+B \Phi_{\varepsilon}^{\prime}\left(g_{\varepsilon}^{n}\right) \partial_{t} g_{\varepsilon}^{n} \mathrm{~d} x \\
= & \int_{0}^{L} a_{\varepsilon}\left(f_{\varepsilon}^{n}\right) \partial_{x}^{3}\left(A f_{\varepsilon}^{n}+B g_{\varepsilon}^{n}\right) \sum_{k=0}^{n}\left(\Phi_{\varepsilon}^{\prime}\left(f_{\varepsilon}^{n}\right) \mid \phi_{k}\right) \partial_{x} \phi_{k} \\
& +B a_{\varepsilon}\left(g_{\varepsilon}^{n}\right) \partial_{x}^{3}\left(f_{\varepsilon}^{n}+g_{\varepsilon}^{n}\right) \sum_{k=0}^{n}\left(\Phi_{\varepsilon}^{\prime}\left(g_{\varepsilon}^{n}\right) \mid \phi_{k}\right) \partial_{x} \phi_{k} \mathrm{~d} x
\end{aligned}
$$

and, integrating with respect to time, we arrive at

$$
\begin{gathered}
\int_{0}^{L} \Phi_{\varepsilon}\left(f_{\varepsilon}^{n}(T)\right)+B \Phi_{\varepsilon}\left(g_{\varepsilon}^{n}(T)\right) \mathrm{d} x \\
=\int_{Q_{T}}\left[a_{\varepsilon}\left(f_{\varepsilon}^{n}\right)\left(A \partial_{x}^{3} f_{\varepsilon}^{n}+B \partial_{x}^{3} g_{\varepsilon}^{n}\right) \sum_{k=0}^{n}\left(\Phi_{\varepsilon}^{\prime}\left(f_{\varepsilon}^{n}\right) \mid \phi_{k}\right) \partial_{x} \phi_{k}\right. \\
\left.+B a_{\varepsilon}\left(g_{\varepsilon}^{n}\right)\left(\partial_{x}^{3} f_{\varepsilon}^{n}+\partial_{x}^{3} g_{\varepsilon}^{n}\right) \sum_{k=0}^{n}\left(\Phi_{\varepsilon}^{\prime}\left(g_{\varepsilon}^{n}\right) \mid \phi_{k}\right) \partial_{x} \phi_{k}\right] \mathrm{d} x \mathrm{~d} t \\
+\int_{0}^{L} \Phi_{\varepsilon}\left(f_{0}^{n}\right)+B \Phi_{\varepsilon}\left(g_{0}^{n}\right) \mathrm{d} x .
\end{gathered}
$$


In order to pass to the limit $n \rightarrow \infty$ in relation (2.21) we must determine what happens with the two integrals on the right-hand side of (2.21). Using (2.19), we obtain

$$
\begin{aligned}
& \left\|\sum_{k=0}^{n}\left(\Phi_{\varepsilon}^{\prime}\left(f_{\varepsilon}^{n}\right) \mid \phi_{k}\right) \partial_{x} \phi_{k}-\Phi_{\varepsilon}^{\prime \prime}\left(f_{\varepsilon}\right) \partial_{x} f_{\varepsilon}\right\|_{L_{2}\left(Q_{T}\right)}^{2} \\
& \leqslant 2\left\|\sum_{k=0}^{n}\left(\Phi_{\varepsilon}^{\prime}\left(f_{\varepsilon}^{n}\right)-\Phi_{\varepsilon}^{\prime}\left(f_{\varepsilon}\right) \mid \phi_{k}\right) \partial_{x} \phi_{k}\right\|_{L_{2}\left(Q_{T}\right)}^{2} \\
& \quad+2\left\|\sum_{k=0}^{n}\left(\Phi_{\varepsilon}^{\prime}\left(f_{\varepsilon}\right) \mid \phi_{k}\right) \partial_{x} \phi_{k}-\Phi_{\varepsilon}^{\prime \prime}\left(f_{\varepsilon}\right) \partial_{x} f_{\varepsilon}\right\|_{L_{2}\left(Q_{T}\right)}^{2} .
\end{aligned}
$$

Taking into account that the first sum on the right-hand side of the above inequality is the truncation of the Fourier series of $\Phi_{\varepsilon}^{\prime \prime}\left(f_{\varepsilon}^{n}\right) \partial_{x} f_{\varepsilon}^{n}-\Phi_{\varepsilon}^{\prime \prime}\left(f_{\varepsilon}\right) \partial_{x} f_{\varepsilon}$ (cf. (2.19)), its norm may be estimated as follows:

$$
\begin{gathered}
\| \sum_{k=0}^{n}\left(\Phi_{\varepsilon}^{\prime}\left(f_{\varepsilon}^{n}\right)-\right. \\
\left.\Phi_{\varepsilon}^{\prime}\left(f_{\varepsilon}\right) \mid \phi_{k}\right) \partial_{x} \phi_{k} \|_{L_{2}\left(Q_{T}\right)}^{2} \\
\leqslant\left\|\Phi_{\varepsilon}^{\prime \prime}\left(f_{\varepsilon}^{n}\right) \partial_{x} f_{\varepsilon}^{n}-\Phi_{\varepsilon}^{\prime \prime}\left(f_{\varepsilon}\right) \partial_{x} f_{\varepsilon}\right\|_{L_{2}\left(Q_{T}\right)}^{2} \\
\leqslant 2\left\|\Phi_{\varepsilon}^{\prime \prime}\left(f_{\varepsilon}^{n}\right)-\Phi_{\varepsilon}^{\prime \prime}\left(f_{\varepsilon}\right)\right\|_{L_{\infty}\left(Q_{T}\right)}^{2}\left\|\partial_{x} f_{\varepsilon}\right\|_{L_{2}\left(Q_{T}\right)}^{2} \\
+2\left\|\Phi_{\varepsilon}^{\prime \prime}\left(f_{\varepsilon}^{n}\right)\right\|_{L_{\infty}\left(Q_{T}\right)}^{2}\left\|\partial_{x} f_{\varepsilon}^{n}-\partial_{x} f_{\varepsilon}\right\|_{L_{2}\left(Q_{T}\right)}^{2} \\
\leqslant 2 \varepsilon^{-4}\left\|f_{\varepsilon}^{n}-f_{\varepsilon}\right\|_{L_{\infty}\left(Q_{T}\right)}^{2}\left\|\partial_{x} f_{\varepsilon}\right\|_{L_{2}\left(Q_{T}\right)}^{2} \\
+2 \varepsilon^{-2}\left\|\partial_{x} f_{\varepsilon}^{n}-\partial_{x} f_{\varepsilon}\right\|_{L_{2}\left(Q_{T}\right)}^{2} .
\end{gathered}
$$

We note that the last inequality has been obtained by using the fact that $\Phi_{\varepsilon}^{\prime \prime}$ is Lipschitz continuous with Lipschitz constant $\varepsilon^{-2}$ and $0 \leqslant \Phi_{\varepsilon}^{\prime \prime} \leqslant \varepsilon^{-1}$, properties which readily follow from $(2.1),(2.2)$, and the relation $\Phi_{\varepsilon}^{\prime \prime}=1 / a_{\varepsilon}$. Invoking (2.16), we resume our calculation with

$$
\left\|\sum_{k=0}^{n}\left(\Phi_{\varepsilon}^{\prime}\left(f_{\varepsilon}^{n}\right)-\Phi_{\varepsilon}^{\prime}\left(f_{\varepsilon}\right) \mid \phi_{k}\right) \partial_{x} \phi_{k}\right\|_{L_{2}\left(Q_{T}\right)}^{2} \underset{n \rightarrow \infty}{\longrightarrow} 0 .
$$

Concerning the second term, we obtain from (2.19) that

$$
\left\|\sum_{k=0}^{n}\left(\Phi_{\varepsilon}^{\prime}\left(f_{\varepsilon}\right) \mid \phi_{k}\right) \partial_{x} \phi_{k}-\Phi_{\varepsilon}^{\prime \prime}\left(f_{\varepsilon}\right) \partial_{x} f_{\varepsilon}\right\|_{L_{2}}=\left\|\sum_{k=n+1}^{\infty}\left(\Phi_{\varepsilon}^{\prime}\left(f_{\varepsilon}\right) \mid \phi_{k}\right) \partial_{x} \phi_{k}\right\|_{L_{2}} \searrow_{n \rightarrow \infty} 0
$$

for almost all $t \in[0, T]$, and Lebesgue's dominated convergence theorem yields

$$
\left\|\sum_{k=0}^{n}\left(\Phi_{\varepsilon}^{\prime}\left(f_{\varepsilon}^{n}\right) \mid \phi_{k}\right) \partial_{x} \phi_{k}-\Phi_{\varepsilon}^{\prime \prime}\left(f_{\varepsilon}\right) \partial_{x} f_{\varepsilon}\right\|_{L_{2}\left(Q_{T}\right)} \underset{n \rightarrow \infty}{\longrightarrow} 0 .
$$

Gathering (2.22) and (2.23), we conclude that

$$
\sum_{k=0}^{n}\left(\Phi_{\varepsilon}^{\prime}\left(f_{\varepsilon}^{n}\right) \mid \phi_{k}\right) \partial_{x} \phi_{k} \underset{n \rightarrow \infty}{\longrightarrow} \Phi_{\varepsilon}^{\prime \prime}\left(f_{\varepsilon}\right) \partial_{x} f_{\varepsilon} \quad \text { in } L_{2}\left(Q_{T}\right) .
$$


Clearly, (2.24) remains true if we replace $f$ by $g$. We sum (2.18), (2.24), use (2.16) and the fact that both $f_{0}$ and $g_{0}$ are non-negative to obtain from (2.21), when letting $n \rightarrow \infty$, the desired assertion (2.4b).

\section{The proof of theorem 1.1}

We shall use the global weak solutions $\left(f_{\varepsilon}, g_{\varepsilon}\right)$ of the regularized problem (2.3) to find, in the limit $\varepsilon \rightarrow 0$, global weak solutions of our original system (1.1). The key role is now played by the second energy functional $\mathcal{E}_{2}$, which will be used to prove that the weak solutions we obtain are non-negative and to identify in $L_{2}\left(0, T ; H^{2}\right)$ a weak limit of the global solutions of (2.3). Using integration by parts, we may then eliminate from the right-hand side of proposition 2.1(ii) the third-order derivatives of $f_{\varepsilon}$ and $g_{\varepsilon}$, for which we do not have any kind of uniform bounds, and obtain assertion (iii) of theorem 1.1 in the limit $\varepsilon \rightarrow 0$.

To do so, we collect first some estimates for the family $\left(f_{\varepsilon}, g_{\varepsilon}\right)$ which were established in $\S 2$. We must pay attention because some of the estimates proven before are uniform only with respect to $n$ and of no use in this final part. Invoking (2.10) and (2.13), we deduce the following:

$f_{\varepsilon}, g_{\varepsilon}$ are uniformly bounded in $L_{\infty}\left(0, T ; H^{1}\right)$,

$\sqrt{a_{\varepsilon}\left(f_{\varepsilon}\right)} \partial_{x}^{3}\left(A f_{\varepsilon}+B g_{\varepsilon}\right), \sqrt{a_{\varepsilon}\left(g_{\varepsilon}\right)} \partial_{x}^{3}\left(f_{\varepsilon}+g_{\varepsilon}\right)$ are uniformly bounded in $L_{2}\left(Q_{T}\right)$,

while, by virtue of $(2.4 a)$ and $(2.4 b)$, we have

$$
\begin{gathered}
\partial_{x}^{2} f_{\varepsilon}, \partial_{x}^{2} g_{\varepsilon} \quad \text { are uniformly bounded in } L_{2}\left(Q_{T}\right), \\
\int_{0}^{L} f_{\varepsilon}(T) \mathrm{d} x=\left\|f_{0}\right\|_{L_{1}}, \quad \int_{0}^{L} g_{\varepsilon}(T) \mathrm{d} x=\left\|g_{0}\right\|_{L_{1}},
\end{gathered}
$$

for all $T>0$. Lastly, we observe that the estimates (2.13) and (2.15) are both uniform with respect to $\varepsilon \in(0,1]$ and $n \in \mathbb{N}$. This implies that the families $\left\{f_{\varepsilon}^{n}: \varepsilon \in(0,1], n \in \mathbb{N}\right\}$ and $\left\{g_{\varepsilon}^{n}: \varepsilon \in(0,1], n \in \mathbb{N}\right\}$ are both relatively compact in $C\left([0, T], C^{\alpha}([0, L])\right)$, if $\alpha \in\left[0, \frac{1}{2}\right)$, and therefore

$$
\left(f_{\varepsilon}\right),\left(g_{\varepsilon}\right) \text { are relatively compact in } C\left([0, T], C^{\alpha}([0, L])\right) \text {. }
$$

Consequently, there exist subsequences $\left(f_{\varepsilon_{k}}\right)$ and $\left(g_{\varepsilon_{k}}\right)$ and functions $f, g$ such that

$$
f_{\varepsilon_{k}} \rightarrow f \quad \text { and } \quad g_{\varepsilon_{k}} \rightarrow g \quad \text { in } C\left([0, T], C^{\alpha}([0, L])\right),
$$

while, owing to (3.1), (3.3), we conclude that $f_{\varepsilon}, g_{\varepsilon}$ are bounded in $L_{2}\left(0, T ; H^{2}\right)$, which ensures, after possibly extracting further subsequences, weak convergence in $L_{2}\left(Q_{T}\right)$ of the spatial derivatives up to order 2:

$$
\partial_{x}^{p} f_{\varepsilon_{k}} \rightarrow \partial_{x}^{p} f \quad \text { and } \quad \partial_{x}^{p} g_{\varepsilon_{k}} \rightarrow \partial_{x}^{p} g \quad \text { in } L_{2}\left(Q_{T}\right) \text { for } p=1,2 .
$$

Recalling proposition 2.1(i) and (3.6), we deduce that $f, g \in L_{2}\left(0, T ; H_{\Delta}^{2}\right)$ for all $T>0$. Moreover, the sequences $\left(f_{\varepsilon_{k}}\right)$ and $\left(g_{\varepsilon_{k}}\right)$ converge strongly towards $f$ and $g$, respectively, in a different norm than in (3.6). 
Lemma 3.1. Given $T>0$, we have

$$
f_{\varepsilon_{k}} \rightarrow f \quad \text { and } \quad g_{\varepsilon_{k}} \rightarrow g \text { in } L_{4}\left(0, T ; H^{1}\right) .
$$

Proof. We prove only the assertion for $f$. Since $f(t)$ and $f_{\varepsilon_{k}}(t)$ belong to $H_{\Delta}^{2}$ for almost all $t \in[0, T]$, we conclude that their first-order derivatives at 0 and $L$ must vanish. Whence, using integration by parts, we get

$$
\begin{aligned}
\int_{0}^{T}\left(\int_{0}^{L}\left|\partial_{x}\left(f_{\varepsilon_{k}}-f\right)\right|^{2} \mathrm{~d} x\right)^{2} \mathrm{~d} t & =\int_{0}^{T}\left(\int_{0}^{L} \partial_{x}^{2}\left(f_{\varepsilon_{k}}-f\right)\left(f_{\varepsilon_{k}}-f\right) \mathrm{d} x\right)^{2} \mathrm{~d} t \\
& \leqslant \int_{0}^{T}\left\|\partial_{x}^{2}\left(f_{\varepsilon_{k}}-f\right)\right\|_{L_{2}}^{2}\left\|f_{\varepsilon_{k}}-f\right\|_{L_{2}}^{2} \mathrm{~d} t \\
& \leqslant L\left\|f_{\varepsilon_{k}}-f\right\|_{L_{\infty}\left(Q_{T}\right)}^{2}\left\|\partial_{x}^{2}\left(f_{\varepsilon_{k}}-f\right)\right\|_{L_{2}\left(Q_{T}\right)}^{2}
\end{aligned}
$$

and, together with (3.3) and (3.6), we get the desired conclusion.

In particular, we obtain from (3.8) that $f_{\varepsilon_{k}}(t) \rightarrow f(t)$ and $g_{\varepsilon_{k}}(t) \rightarrow g(t)$ in $H^{1}$ for almost all $t \in[0, T]$, and, together with the estimate (3.1), we conclude that $f, g \in L_{\infty}\left(0, T ; H^{1}\right)$. Furthermore, $f_{\varepsilon}(0)=f_{0}$ and $g_{\varepsilon}(0)=g_{0}$ for all $\varepsilon \in(0,1]$, so that (3.6) yields $f(0)=f_{0}$ and $g(0)=g_{0}$. The estimate of theorem 1.1(iv) follows by combining (3.6) with assertion $(2.4 a)$ and lemma 3.2, below.

We now use the energy estimate $(2.4 b)$ to establish assertion (i) of our main result, theorem 1.1.

Lemma 3.2. The functions $f$ and $g$ found above are non-negative.

Proof. Assume that there exists $\left(T, x_{0}\right) \in Q_{\infty}$ such that $f\left(T, x_{0}\right)<0$. Since, by (3.6), $f_{\varepsilon_{k}} \rightarrow f$ in $C\left(\bar{Q}_{T}\right)$, we conclude that there exists a constant $\delta>0$ and $k_{0} \in \mathbb{N}$ with the property that $f_{\varepsilon_{k}}(T, x)<-\delta$ for all $x \in[0, L]$ with $\left|x-x_{0}\right|<\delta$ and all $k \geqslant k_{0}$. We then infer from (2.2) that

$$
\Phi_{\varepsilon_{k}}\left(f_{\varepsilon_{k}}(T, x)\right)=\frac{f_{\varepsilon_{k}}^{2}(T, x)}{2 \varepsilon_{k}}+f_{\varepsilon_{k}}(T, x) \ln \left(\varepsilon_{k}\right)+\varepsilon_{k} \ln \left(\varepsilon_{k}\right)-\varepsilon_{k}+1 \geqslant \frac{\delta^{2}}{2 \varepsilon_{k}}
$$

for all $x$ and $k$ as above. This contradicts the assertion (2.4b). Clearly, the argument is true when replacing $f$ by $g$, and this proves the claim.

In order to deduce the energy estimate theorem 1.1(v), we recall (2.2) and note that, for all $k \in \mathbb{N}$, we have $\Phi_{\varepsilon_{k}}\left(f_{\varepsilon_{k}}\right) \geqslant \tilde{\Phi}_{\varepsilon_{k}}\left(f_{\varepsilon_{k}}\right)$, where

$$
\tilde{\Phi}_{\varepsilon_{k}}(s):= \begin{cases}\left(s+\varepsilon_{k}\right) \ln \left(s+\varepsilon_{k}\right)-\left(s+\varepsilon_{k}\right)+1, & s \geqslant 0, \\ \varepsilon_{k} \ln \left(\varepsilon_{k}\right)-\varepsilon_{k}+1, & s<0 .\end{cases}
$$

Given $t \in[0, T]$, the sequence $\left(\tilde{\Phi}_{\varepsilon_{k}}\left(f_{\varepsilon_{k}}(t)\right)\right)$ is bounded in $C([0, L])$ and

$$
\tilde{\Phi}_{\varepsilon_{k}}\left(f_{\varepsilon_{k}}(t)\right) \rightarrow \Phi(f(t))
$$

pointwise on $[0, L]$. Lebesgue's dominated convergence then implies

$$
\liminf _{k \rightarrow \infty} \int_{0}^{L} \Phi_{\varepsilon_{k}}\left(f_{\varepsilon_{k}}(T)\right) \mathrm{d} x \geqslant \liminf _{k \rightarrow \infty} \int_{0}^{L} \tilde{\Phi}_{\varepsilon_{k}}\left(f_{\varepsilon_{k}}(T)\right) \mathrm{d} x=\int_{0}^{L} \Phi(f(T)) \mathrm{d} x .
$$


Of course, the relation still remains true when replacing $f$ by $g$. By virtue of (3.7), we may pass to $\liminf \operatorname{in}_{k \rightarrow \infty}$ in relation $(2.4 b)$, and obtain in this way the desired energy estimate $(\mathrm{v})$ of theorem 1.1 .

To deal with the energy estimate (vi) of theorem 1.1, we observe first that, for all $k \in \mathbb{N}$,

$$
\left|a_{\varepsilon_{k}}\left(f_{\varepsilon_{k}}\right)-f\right| \leqslant \varepsilon_{k}+\left|f_{\varepsilon_{k}}-f\right|
$$

meaning, by (3.6), that

$$
a_{\varepsilon_{k}}\left(f_{\varepsilon_{k}}\right) \rightarrow f \quad \text { and } \quad a_{\varepsilon_{k}}\left(g_{\varepsilon_{k}}\right) \rightarrow g \text { in } C\left(\bar{Q}_{T}\right) .
$$

For every positive integer $m$, we now introduce the set

$$
Q_{T}^{m}:=\left\{(t, x) \in Q_{T}: f(t, x)>m^{-1} \text { and } g(t, x)>m^{-1}\right\},
$$

where we may control, by virtue of the estimate $(2.4 c)$ and (3.10), the third-order derivatives of both $f_{\varepsilon_{k}}$ and $g_{\varepsilon_{k}}$ :

$$
\left(\partial_{x}^{3} f_{\varepsilon_{k}}\right),\left(\partial_{x}^{3} g_{\varepsilon_{k}}\right) \text { are uniformly bounded in } L_{2}\left(Q_{T}^{m}\right) .
$$

Taking into account that $Q_{T}^{+}=\bigcup_{m} Q_{T}^{m}$, we may assume, after possibly extracting a further subsequence, that

$$
\partial_{x}^{3} f_{\varepsilon_{k}} \rightarrow \partial_{x}^{3} f, \quad \partial_{x}^{3} g_{\varepsilon_{k}} \rightarrow \partial_{x}^{3} g \quad \text { in } L_{2}\left(Q_{T}^{m}\right)
$$

for all $m \in \mathbb{N}$, which, together with (3.10), implies

$$
\left.\begin{array}{c}
\sqrt{a_{\varepsilon_{k}}\left(f_{\varepsilon_{k}}\right)} \partial_{x}^{3}\left(A f_{\varepsilon_{k}}+B g_{\varepsilon_{k}}\right) \rightarrow \sqrt{f} \partial_{x}^{3}(A f+B g), \\
\sqrt{a_{\varepsilon_{k}}\left(g_{\varepsilon_{k}}\right)} \partial_{x}^{3}\left(f_{\varepsilon_{k}}+g_{\varepsilon_{k}}\right) \rightarrow \sqrt{g} \partial_{x}^{3}(f+g)
\end{array}\right\} \quad \text { in } L_{1}\left(Q_{T}^{m}\right) .
$$

In fact, by virtue of (3.2), the weak convergence in (3.12) takes place in $L_{2}\left(Q_{T}^{m}\right)$. Recalling (2.4c) and lemma 3.1, for $k \rightarrow \infty$, we obtain the desired estimates (ii) and (vi) of theorem 1.1.

In order to complete the proof of theorem 1.1, we are left to prove the relations (iii). To this end, we pick $\psi \in H_{\Delta}^{2}$. Since $a_{\varepsilon_{k}}$ is Lipschitz continuous, we obtain from proposition 2.1(i) that $a_{\varepsilon_{k}}\left(f_{\varepsilon_{k}}(t)\right) \in H^{1}$ for almost all $t \in(0, T)$ and

$$
\partial_{x}\left(a_{\varepsilon_{k}}\left(f_{\varepsilon_{k}}\right)\right)(t, x)=\chi_{(0, \infty)}\left(f_{\varepsilon_{k}}\right) \partial_{x} f_{\varepsilon_{k}} \quad \text { almost everywhere in } Q_{T},
$$

whereby $\chi_{(0, \infty)}$ denotes the characteristic function of the interval $(0, \infty)$. Integrating by parts in the first relation of proposition 2.1 (ii), we arrive at

$$
\begin{aligned}
\int_{0}^{L} f_{\varepsilon_{k}}(T) \psi \mathrm{d} x-\int_{0}^{L} f_{0} \psi \mathrm{d} x & =\int_{0}^{T} \int_{0}^{L} a_{\varepsilon_{k}}\left(f_{\varepsilon_{k}}\right) \partial_{x}^{3}\left(A f_{\varepsilon_{k}}+B g_{\varepsilon_{k}}\right) \partial_{x} \psi \mathrm{d} x \mathrm{~d} t \\
& =I_{1, k}+I_{2, k}
\end{aligned}
$$

where

$$
\begin{aligned}
& I_{1, k}:=-\int_{Q_{T}} \partial_{x}\left(a_{\varepsilon_{k}}\left(f_{\varepsilon_{k}}\right)\right) \partial_{x}^{2}\left(A f_{\varepsilon_{k}}+B g_{\varepsilon_{k}}\right) \partial_{x} \psi \mathrm{d} x \mathrm{~d} t \\
& I_{2, k}:=-\int_{Q_{T}} a_{\varepsilon_{k}}\left(f_{\varepsilon_{k}}\right) \partial_{x}^{2}\left(A f_{\varepsilon_{k}}+B g_{\varepsilon_{k}}\right) \partial_{x}^{2} \psi \mathrm{d} x \mathrm{~d} t
\end{aligned}
$$


We note the use of $\psi \in H_{\Delta}^{2}$ to eliminate the boundary terms in (3.13) due to $\partial_{x} \psi(0)=\partial_{x} \psi(L)=0$.

Combining (3.7) and (3.10), we obtain for $k \rightarrow \infty$ that

$$
I_{2, k} \rightarrow-\int_{Q_{T}} f\left(A \partial_{x}^{2} f+B \partial_{x}^{2} g\right) \partial_{x}^{2} \psi \mathrm{d} x \mathrm{~d} t .
$$

We consider now the integral $I_{1, k}$, and note that in order to show the relation

$$
I_{1, k} \rightarrow-\int_{Q_{T}} \partial_{x} f\left(A \partial_{x}^{2} f+B \partial_{x}^{2} g\right) \partial_{x} \psi \mathrm{d} x \mathrm{~d} t
$$

it suffices to prove that

$$
\partial_{x}\left(a_{\varepsilon_{k}}\left(f_{\varepsilon_{k}}\right)\right) \rightarrow \partial_{x} f \quad \text { in } L_{2}\left(Q_{T}\right) .
$$

To this end, we write

$$
\partial_{x}\left(a_{\varepsilon_{k}}\left(f_{\varepsilon_{k}}\right)\right)-\partial_{x} f=\left(\partial_{x}\left(a_{\varepsilon_{k}}\left(f_{\varepsilon_{k}}\right)\right)-\partial_{x} f_{\varepsilon_{k}}\right)+\left(\partial_{x} f_{\varepsilon_{k}}-\partial_{x} f\right),
$$

and conclude from lemma 3.1 that $\left(\partial_{x} f_{\varepsilon_{k}}-\partial_{x} f\right) \rightarrow 0$ in $L_{2}\left(Q_{T}\right)$. Furthermore, the first term may be written as

$$
\left(\partial_{x}\left(a_{\varepsilon_{k}}\left(f_{\varepsilon_{k}}\right)\right)-\partial_{x} f_{\varepsilon_{k}}\right)=\left(\chi_{(0, \infty)}\left(f_{\varepsilon_{k}}\right)-1\right) \partial_{x} f_{\varepsilon_{k}},
$$

and since $\partial_{x} f_{\varepsilon_{k}} \rightarrow \partial_{x} f$ in $L_{2}\left(Q_{T}\right)$, there exists a function $F \in L_{2}\left(Q_{T}\right)$ such that, after possibly extracting a further subsequence, $\left|\partial_{x} f_{\varepsilon_{k}}\right| \leqslant F$ almost everywhere in $Q_{T}$ (see the proof of [12, Theorem 3.11]). We show now that

$$
\left(\chi_{(0, \infty)}\left(f_{\varepsilon_{k}}\right)-1\right) \partial_{x} f_{\varepsilon_{k}} \rightarrow 0
$$

almost everywhere in $Q_{T}$. Indeed, since $\partial_{x} f_{\varepsilon_{k}} \rightarrow \partial_{x} f$ in $L_{2}\left(Q_{T}\right)$, we deduce that $\partial_{x} f_{\varepsilon_{k}} \rightarrow 0$ almost everywhere on the set $[f=0]$. Furthermore, on the set $[f>0]$, relation (3.6) implies pointwise convergence $\left(\chi_{(0, \infty)}\left(f_{\varepsilon_{k}}\right)-1\right) \rightarrow 0$. Lebesgue's dominated convergence theorem now implies the desired relation (3.16), and, implicitly, (3.15).

To conclude, we sum (3.6), (3.14) and (3.15) and let $k \rightarrow \infty$ in relation (3.13) to obtain the first identity of theorem 1.1(iii). The corresponding relation for $g$ follows similarly.

\section{Acknowledgements}

The author thanks Philippe Laurençot for deep and fruitful discussions.

\section{References}

1 F. Bernis. Viscous flows, fourth-order nonlinear degenerate parabolic equations and singular elliptic problems. In Free boundary problems: theory and applications (ed. J. Diaz, M. Herrero, A. Liñan and J. Vazquez), Pitman Research Notes in Mathematics, vol. 323, pp. 400-56 (Pitman, 1995).

2 F. Bernis and A. Friedman. Higher order nonlinear degenerate parabolic equations. J. Diff. Eqns 83 (1990), 179-206. 
3 A. L. Bertozzi and M. Pugh. The lubrication approximation for thin viscous films: the moving contact line with a 'porous media' cut-off of van der Waals interactions. Nonlinearity 7 (1994), 1535-1564.

4 A. L. Bertozzi and M. Pugh. The lubrication approximation for thin viscous films: regularity and long-time behaviour of weak solutions. Commun. Pure Appl. Math. 49 (1996), 85-123.

5 R. Dal Passo, H. Garcke, and G. Grün. On a fourth-order degenerate parabolic equation: global entropy estimates, existence, and qualitative behavior of solutions. SIAM J. Math. Analysis 29 (1998), 321-342.

6 J. Escher, Ph. Laurençot and B.-V. Matioc. Existence and stability of weak solutions for a degenerate parabolic system modelling two-phase flows in porous media. Annales Inst. H. Poincaré Analyse Non Linéaire 28 (2011), 583-598.

7 J. Escher, A.-V. Matioc and B.-V. Matioc. Modelling and analysis of the Muskat problem for thin fluid layers. J. Math. Fluid Mech. 14 (2012), 267-277.

8 J. Escher, A.-V. Matioc and B.-V. Matioc. A generalized Rayleigh-Taylor condition for the Muskat problem. Nonlinearity 25 (2012), 73-92.

9 M. Günther and G. Prokert. A justification for the thin film approximation of Stokes flow with surface tension. J. Diff. Eqns 245 (2008), 2802-2845.

10 J. Hulshof. Some aspects of the thin film equation. In Proc. Europ. Congr. of Mathematics, Vol. II, pp. 291-301 (Birkhäuser 2001).

11 B.-V. Matioc and G. Prokert. Hele-Shaw flow in thin threads: a rigorous limit result. Interfaces Free Bound. 14 (2012), 205-230.

12 W. Rudin. Real and complex analysis (McGraw-Hill, 1987).

13 J. Simon. Compact sets in the space $L^{p}(0, T ; B)$. Annali Mat. Pura Appl. 4(146) (1987), 65-96.

14 A. Tudorascu. Lubrication approximation for thin viscous films: asymptotic behaviour of nonnegative solutions. Commun. PDEs 32 (2007), 1147-1172.

(Issued 5 October 2012) 